\title{
Effects of long-term cadmium exposure on urinary metabolite profiles in mice
}

\author{
Sailendra Nath Sarma'1, Ammar Saleem¹, Jin-Yong Lee², Maki Tokumoto², \\ Gi-Wook Hwang ${ }^{3}$, Hing Man Chan ${ }^{1}$ and Masahiko Satoh ${ }^{2}$ \\ ${ }^{1}$ Department of Biology, University of Ottawa, Ottawa, Ontario K1N 6N5, Canada \\ ${ }^{2}$ Laboratory of Pharmaceutical Health Sciences, School of Pharmacy, Aichi Gakuin University, \\ 1-100 Kusumoto-cho, Chikusa-ku, Nagoya 464-8650, Japan \\ ${ }^{3}$ Laboratory of Molecular Biochemical Toxicology, Graduate School of Pharmaceutical Sciences, Tohoku University, \\ Aoba-ku, Sendai 980-8578, Japan
}

(Received September 11, 2017; Accepted December 5, 2017)

\begin{abstract}
Cadmium (Cd) is a common environmental pollutant with known toxic effects on the kidney. Urinary metabolomics is a promising approach to study mechanism by which Cd-induced nephrotoxicity. The aim of this study was to elucidate the mechanism of $\mathrm{Cd}$ toxicity and to develop specific biomarkers by identifying urinary metabolic changes after a long-term of $\mathrm{Cd}$ exposure and with the critical concentration of $\mathrm{Cd}$ in the kidney. Urine samples were collected from wild-type $129 / \mathrm{Sv}$ mice after 67 weeks of $300 \mathrm{ppm} \mathrm{Cd}$ exposure and analyzed by ultra performance liquid chromatography connected with quadrupole time of flight mass spectrometer (UPLC-QTOF-MS) based metabolomics approach. A total of 40 most differentiated metabolites ( 9 down-regulated and 31 up-regulated) between the control and $\mathrm{Cd}$-exposed group were identified. The majority of the regulated metabolites are amino acids (glutamine, $L$-aspartic acid, phenylalanine, tryptophan, and $D$-proline) indicating that amino acid metabolism pathways are affected by long-term exposure of $\mathrm{Cd}$. However, there are also some nucleotides (guanosine, guanosine monophosphate, cyclic AMP, uridine), amino acid derivatives (homoserine, $N$-acetyl- $L$-aspartate, $N$-acetylglutamine, acetyl-phenylalanine, carboxymethyllysine), and peptides. Results of pathway analysis showed that the arginine and proline metabolism, purine metabolism, alanine, aspartate and glutamate metabolism, and aminoacyl-tRNA biosynthesis were affected compared to the control. This study demonstrates that metabolomics is useful to elucidate the metabolic responses and biological effects induced by Cd-exposure.
\end{abstract}

Key words: Cadmium, Kidney, Long-term exposure, Urine metabolome

\section{INTRODUCTION}

Humans are exposed to cadmium (Cd) from the diet, cigarette smoke, and environmental and occupational sources (Fowler, 2009). Cd is not readily excreted resulting in a long half-life of 10-35 years in humans (Peters et al., 2010). Previous studies demonstrated that low level of Cd exposure caused the kidney toxicity (Ginsberg, 2012; Järup et al., 2000). In animal studies, long-term Cd exposure from dietary induced injury to mouse kidney (Lee et al., 2016a; Tokumoto et al., 2011). With the long-term exposure, $\mathrm{Cd}$ accumulates in the proximal tubular epithelial cells, where it triggers the re-absorptive dysfunction resulting in polyuria and low molecular weight proteinu- ria (Järup, 2002; Järup and Akesson, 2009; Prozialeck and Edwards, 2010, 2012).

Urinary $\mathrm{Cd}$ concentrations can be used as biomarkers for body burden and proteinuria is commonly used for diagnosis of Cd-induced renal toxicity (Akerstrom et al., 2013). Because urine contains many compounds extracted from the bloodstream or generated by kidney cells themselves, much effort has been devoted to identifying biomarkers in the urine for chronic effects of $\mathrm{Cd}$ exposure on the kidney. For example, some standard metrics such as $\beta_{2}$-microglobulin and $N$-acetyl- $\beta$ - $D$-glucosaminidase (NAG) have been shown to be useful as biomarkers of Cd nephrotoxicity (Halatek et al., 2005; Prozialeck and Edwards, 2012; Prozialeck et al., 2016). However, there

Correspondence: Masahiko Satoh (E-mail: masahiko@dpc.agu.ac.jp) 
are some shortcomings in their applications. For example, NAG is excreted into urine at a relatively late stage of Cd-induced damage (Prozialeck and Edwards, 2012; Prozialeck et al., 2016). $\beta_{2}$-Microglobulin in urinary excretion may also reflect plasma levels of the protein, which can be affected by $\mathrm{Cd}$ at glomerulus or on organs other than the kidney (Halatek et al., 2005; Prozialeck et $a l .$, 2016). Furthermore, their urinary levels are affected by re-absorptive dysfunction, which is caused by proximal tubular cell damage; and other substances can affect their activities (Fels et al., 1998; Lim et al., 2016). Thus, there is a need for better biomarkers that can provide higher specificity for Cd-induced nephrotoxicity.

Non-targeted metabolomics effectively measures downstream genome-wide or proteome-wide interactions of an organism (Forsythe and Wishart, 2009). Metabolite profiling is used to investigate the global endogenous metabolites in the biological systems and its dynamic changes in response to endogenous and exogenous factors (Kaddurah-Daouk et al., 2008). Metabolomics has been shown to be useful for clinical use and research in chronic kidney disease (Hocher and Adamski, 2017). Recent studies have reported the use of urinary metabolomics to identify possible biomarkers and to explain the mechanisms responsible for the pathogenesis and progression of $\mathrm{Cd}$ in rats (Lee et al., 2014), mice (Gong et al., 2017), and in humans (Gao et al., 2014; Xu et al., 2016). It was found that metabolites related to metabolism of mitochondrial energy, amino acid, galactose, purine, intestinal flora, and metabolites involved in creatine pathway, steroid hormone biosynthesis, glutathione biosynthesis pathway, and the tricarboxylic acid cycle were affected by $\mathrm{Cd}$ exposure. In addition, some specific urinary protein-based biomarkers such as osteopontin, monocyte chemoattractant protein-1 (MCP-1), kidney injury molecules-1 (Kim-1), and selenium-binding protein 1 (SBP1) were proposed (Lee et al., 2014).

Majority of the previous animal studies were based on relatively short-term $\mathrm{Cd}$ exposure; however, the similar exposure conditions as human cases were not studied. The objectives of this study were to study the changes in the urinary metabolome of mice exposed to $\mathrm{Cd}$ (300 ppm) over a long period of time (67 weeks). The goal of this study is to identify urinary biomarkers that are specific to Cd toxicity by non-targeted metabolomics approach using an ultra performance liquid chromatography (UPLC) connected with accurate quadrupole time of flight (QTOF) mass spectrometry (MS), UPLC-QTOF-MS.

\section{MATERIALS AND METHODS}

\section{Animals treatment and sample collection}

Wild-type mice of 129/Sv strain (Jackson Laboratory, Bar Harbor, ME, USA) were housed in cages in an animal room at a controlled temperature $24 \pm 2^{\circ} \mathrm{C}$ and relative humidity of $45 \pm 15 \%$, with a $12 \mathrm{hr}$ light/dark cycle. Four-week-old female mice were randomly assigned to control and treatment groups $(n=4-5)$. Control group was access to standard laboratory chow (Oriental Yeast Co., Tokyo, Japan) and water ad libitum. The treatment group was fed 300 ppm Cd containing chow (Oriental Yeast Co.) and water ad libitum. At 67 weeks after $\mathrm{Cd}$ exposure, urine samples were collected for $12 \mathrm{hr}$ from each mouse in urine collection devices. The urine samples were then frozen at $-80^{\circ} \mathrm{C}$ until analysis. All the animal experiments were performed according to Regulation on Animal Experimentation at School of Pharmacy, Aichi Gakuin University.

\section{Cd concentration measurement}

Tissues were digested with nitric acid and hydrogen peroxide. After digestion, inorganic residues were dissolved in ultrapure water, and metal analysis was carried out using atomic absorption spectrometer (200 series AA; Agilent Technologies, Santa Clara, CA, USA).

\section{Renal toxicity evaluation}

To evaluate the renal toxicity, the activity of NAG in the urine was examined using NAG Test Shionogi (Shionogi \& Co. Ltd, Osaka, Japan). NAG activity was determined by the level of products from the reaction of NAG and its substrate using the spectrophotometer $(575 \mathrm{~nm})$. NAG activity was normalized with the creatinine (Cre) level in the urine. The levels of blood urea nitrogen (BUN) in the serum were examined. The automatic dry-chemistry analyzer system (Spotchem EZ SP-4430; Arkray, Kyoto, Japan) was used to determine the BUN and Cre level. The kidney samples were fixed in $10 \%(\mathrm{v} / \mathrm{v})$ neutral buffered formalin solution and embedded in paraffin. Deparaffinized serial tissue sections (thickness, $5 \mu \mathrm{m}$ ) were stained with Hematoxylin and Eosin for histopathological analysis. The pathological image was photographed by EVOS XL system (Life Technologies, Carlsbad, CA, USA).

\section{Sample preparation}

Urine samples were aliquot into 4 portions and lyophilized by Supermodylo freeze dryer (Fisher Scientific, Oakville, ON, Canada). Each aliquot was re-solubilized separately in $1 \mathrm{~mL}$ of in $100 \%$ methanol (solvent system 
1), $100 \%$ water (solvent system 2 ), $40 \%$ methanol $+40 \%$ acetonitrile $+20 \%$ water (solvent system 3), 40\% methanol $+40 \%$ chloroform $+20 \%$ water (solvent system 4). The samples were then filtered through 0.2-micron syringe filters (Chromatographic Specialties, Brockville, ON, Canada).

\section{UPLC-QTOF-MS analysis}

Chromatography was performed on an ACQUITY UPLC BEH C18 column $(1.7 \mu \mathrm{m}, 2.1 \times 100 \mathrm{~mm})$ connected with a VanGuard Pre-column $(2.1 \times 5 \mathrm{~mm})$. ACQUITY UPLC was connected with a Xevo G2 QTOFMS (Waters Inc., Milford, MA, USA). The column thermostat and auto-sampler were maintained at $50^{\circ} \mathrm{C}$ and $4^{\circ} \mathrm{C}$ respectively. The mobile phase consisted of water containing $0.1 \%$ formic acid (A) and acetonitrile containing $0.1 \%$ formic acid (B) (Fisher Optima LC-MS).

Optimized gradient conditions were: mobile phase: 0-1 min 5\% A isocratic, 1-6 min linear gradient, 5-50\% $\mathrm{B}$, 6-8 $\min 50-95 \% \mathrm{~B}, 8-10 \mathrm{~min} 5 \% \mathrm{~A}$ isocratic (total run time $10 \mathrm{~min}$ ). The flow rate was $0.5 \mathrm{~mL} / \mathrm{min}$, and $1 \mu \mathrm{L}$ of sample was injected followed by a strong wash $200 \mu \mathrm{L}(90 \%$ acetonitrile $+10 \%$ water $)$ and weak wash $600 \mu \mathrm{L}(10 \%$ acetonitrile $+90 \%$ water). The performance of ACQUITY system was monitored by triplicate $1 \mu \mathrm{L}$ injections of Waters ACQUITY MS-Start-up solution 1, containing $1 \mu \mathrm{g} / \mathrm{mL}$ of sulfadimethoxine.

QTOF was operated in MS mode. Low-energy and high-energy electrospray spectra were collected in negative and positive ionization modes. Optimized conditions were: source temperature of $120^{\circ} \mathrm{C}$, desolvation temperature of $400^{\circ} \mathrm{C}$, Cone gas $\left(\mathrm{N}_{2}\right)$ flow of $50 \mathrm{~L} / \mathrm{hr}$, and nitrogen desolvation gas flow of $1195 \mathrm{~L} / \mathrm{hr}$. Leucine-enkephalin was used as the lock mass generating a $[\mathrm{M}+\mathrm{H}]^{+}$ion ( $\mathrm{m} / \mathrm{z}$ 556.2615). The optimal conditions used for MS analysis were as follows: mass range 100-1000 Da, function 1 was set at $6 \mathrm{~V}$ to obtain low energy spectra, and function 2 was set at 10-30 V to obtain high energy spectra. Cone voltage $20 \mathrm{~V}$, scan time $0.1 \mathrm{sec}$. The system was calibrated with sodium formate and the data were acquired and processed with MassLynx (version 4.1) and MarkerLynx (version 8.03) software with principal component analysis (PCA). The retention times and the protonated masses were generated at a noise threshold of 500 counts and no smoothing was applied. Because the negative ionization mode was used for data analysis the performance of QTOF was monitored by checking the $[\mathrm{M}-\mathrm{H}]^{-1}(\mathrm{~m} / \mathrm{z}$ 554.1415).

\section{Data Analysis}

Multivariate analyses were performed on MarkerLynx
(Waters Inc.). Unsupervised PCA was carried out by using Pareto scaling to generate the score plots while supervised orthogonal partial least square analyses (OPLS-DA) were performed to identify the up-regulation or down-regulation of the biomarkers. Variables that had significant contributions (i.e., p values $<0.05$ ) between groups were considered as potential biomarkers. Variable importance in projection (VIP) plot was generated to rank the metabolites identified by OPLS-DA. The exact mass and retention time pairs were then plotted to identify discriminant markers.

\section{Metabolite identity}

The accurate masses of the detected metabolites were used to perform elemental composition (elemental composition). These accurate masses and molecular formulae were then used to identify metabolites by matching our in-house spectral library. Further confirmation was done by performing the matches with the masses and molecular formulae endorsed in Metlin (https://metlin.scripps. edu), human metabolome database (http://www.hmdb.ca) and ChemSpider (http://www.chemspider.com) databases by applying the threshold of 5 ppm mass accuracy. Mass fragments, obtained from high energy spectra, were used to confirm the metabolite identity. Standards were not used in this study to confirm the identification hence the identification remains putative. Quantitative enrichment analysis was performed with MetPA (http://metpa.metabolomics.ca) and MSEA software (http://www.metaboanalyst.ca) and compares the molecular content of a metabolic signature with metabolites found in known metabolic pathway maps or databases. MetPA performs pathway analysis and MSEA is the tool to identify biologically meaningful patterns in quantitative metabolomics.

\section{RESULTS}

Body weights of Cd-exposed mice were monitored upon exposure to $\mathrm{Cd}$ for 67 weeks. The body weight of control mice was $29.92 \pm 4.39 \mathrm{~g}$. The body weight of Cdexposed mice was $23.26 \pm 1.14 \mathrm{~g}$, which is significantly lower than that of control mice (Lee et al., 2016b). Cd concentration in the kidney was measured using atomic absorption spectrometer. While Cd was not detected in the kidney of control mice, Cd concentration in the kidney of Cd-exposed mice was $189.54 \pm 12.85 \mu \mathrm{g} / \mathrm{g}$ kidney (Fig. 1A). The Cd concentration, 200 ppm, is thought to be a critical accumulation level in mouse kidney (Bhattacharyya et al., 1988; Friberg, 1984). To determine renal toxicity, the activities of NAG in the urine and the level of BUN in the serum were examined and 

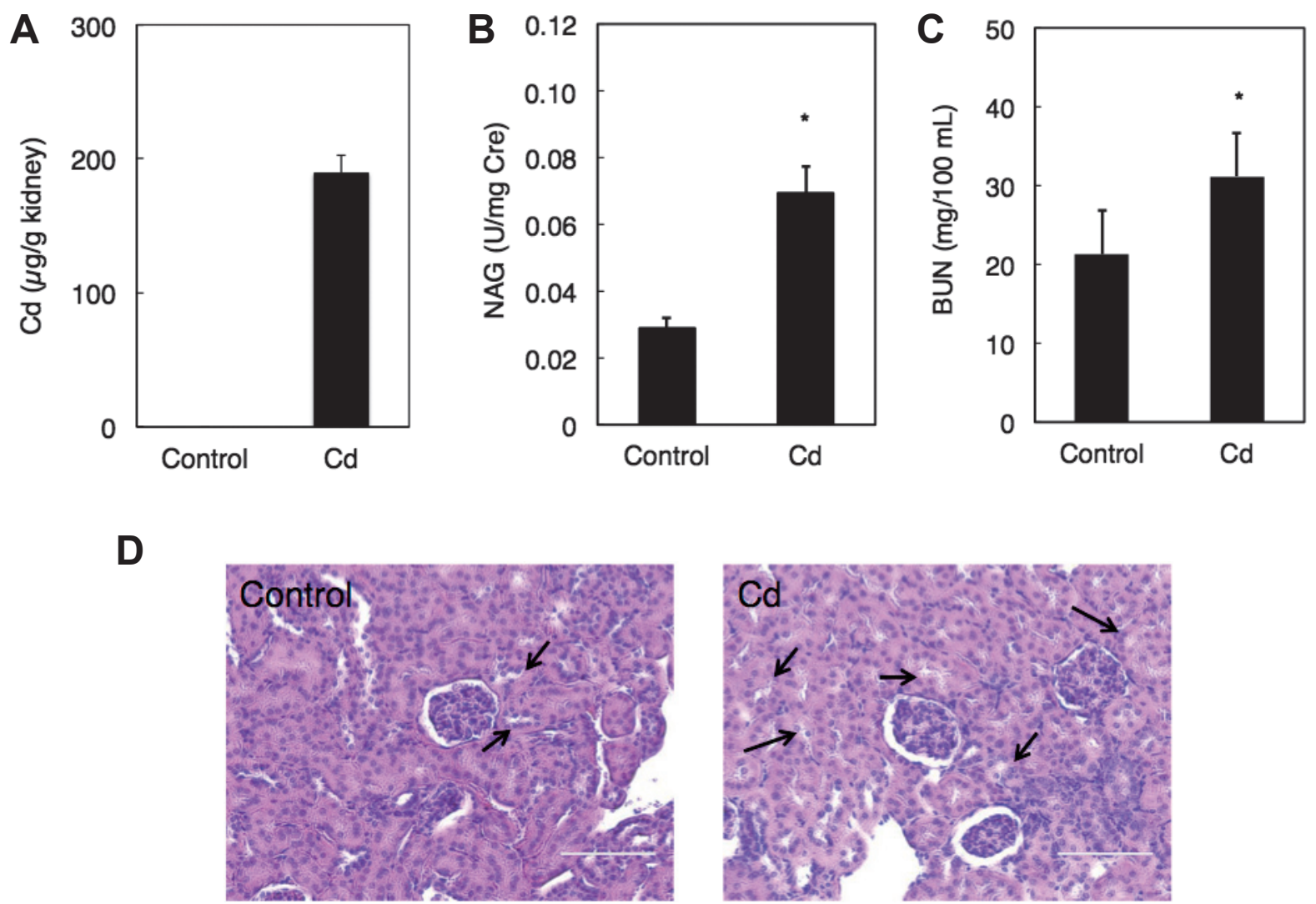

Fig. 1. Renal toxicity of long-term Cd exposure in mice. A. Cd accumulation in the kidney of mice exposed to Cd for 67 weeks. A portion of the kidney was obtained after 67 -week Cd exposure. The kidney was digested with nitric acid and hydrogen peroxide, and the $\mathrm{Cd}$ content was measured by atomic absorption spectrometer. $\mathrm{Cd}$ is not detected in the kidney of control mice. Values are mean \pm S.D. $(\mathrm{n}=4-5)$. *Significantly different from the control group, $P<0.05$. B. NAG activity in the urine. NAG activity was normalized by creatinine (Cre) level in the urine. C. BUN level in the serum. Values are mean \pm S.D. $(\mathrm{n}=4-5)$. *Significantly different from the control group, $P<0.05$. D. Histopathological changes in the kidney of long-term $\mathrm{Cd}$ exposure mice. Kidney was stained with Hematoxyline and Eosin. Arrows mean vacuolations. Scale: $\times 400$.

histopathologic analyses on the kidney were conducted. The activity of NAG in the urine and the level of BUN in the serum of Cd-exposed mice were slightly increased. (Figs. 1B, C). In the kidney section, moderate histologic changes such as vacuolation upon $\mathrm{Cd}$ exposure were observed (Fig. 1D). Slight histologic damage was detected in the kidney of control mice as well (Fig. 1D); it may be due to aging. Therefore, mild renal toxicity was initiated in these long-term $\mathrm{Cd}$ exposure mice. It is almost contemporarily with our previous study using C57BL/6 J strain (Tokumoto et al., 2011).

Pilot studies were conducted to optimize the extraction of metabolites. Our initial results with water or methanol as extraction solvents recovered a very little portion of the metabolome. A solvent system containing $40 \%$ metha- nol $+40 \%$ acetonitrile $+20 \%$ water was found to be optimal, i.e. extracted the maximum number of metabolites $(n=176)$; Figure 2 shows the results of the discriminant analysis (S-plot) of the 176 metabolites. The most discriminant markers/metabolites in the control and Cdexposed group were highlighted and selected for identification. A total of 9 identified metabolites were downregulated and 31 identified metabolites were up-regulated (Fig. 2 and Table 1).

The identity of 40 metabolites based on accurate mass and elemental composition matches in the in-house spectral library within a mass error of $5 \mathrm{ppm}$ (Table 1). The majority of the up-regulated metabolites are amino acids. Down-regulated metabolites include pyrroline hydroxycarboxylic acid that plays a central role in proline arginine 


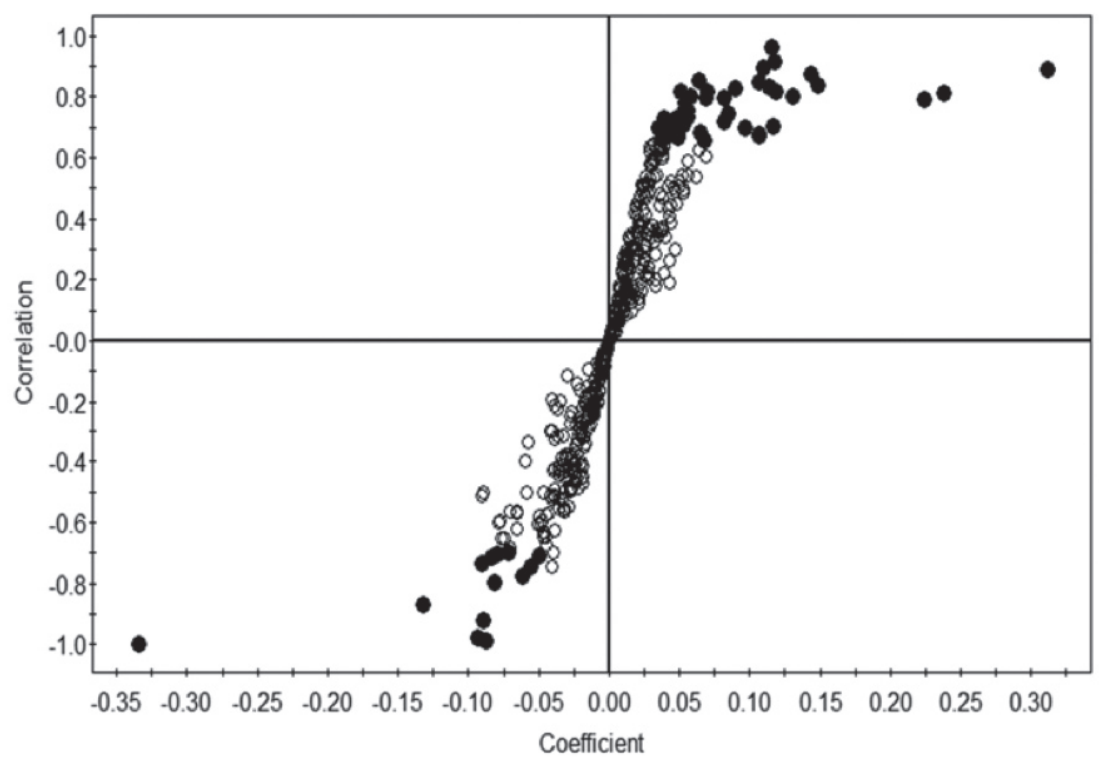

Fig. 2. Discriminant analysis showing the distribution of metabolites in control ( 0.0 to -1.0 , bottom left panel) and Cd exposed $(0.0$ to +1.0 , top right panel). The most discriminative metabolites labeled as dots were selected for identification. Other metabolites labeled as circles were not identified.

metabolism. It is identified in our study as a major downregulated and discriminant marker (Rt $4.77 \mathrm{~min},[\mathrm{M}-\mathrm{H}]^{-1}$, 129.0553). The oxidation of pyrroline hydroxycarboxylic results in the biosynthesis of glutamylcysteine, a major up-regulated metabolite identified in our study (Rt 3.45 $\min ,[\mathrm{M}-\mathrm{H}]^{-1}, 250.0713$ ).

The signaling pathways and molecular networks influenced by $\mathrm{Cd}$ exposure were explored and visualized by MetPA, a web application for metabolomics analysis (Xia and Wishart, 2010a) . Potential biomarkers contributing to the separation of pairwise groups were imported into MetPA. The "Mus musculus" library was selected as the database, while hypergeometric test and relativeness centrality were performed for over-representation analysis and pathway topology analysis, respectively. A list of pathways affected as indicated by the identified metabolites is shown in Table 2. In addition, the graphic outputs are shown in Fig. 3. The nodes with high impact value indicated the most targeted pathways, including arginine and proline metabolism, purine metabolism, and alanine, aspartate and glutamate metabolism pathways. The identified metabolite biomarkers were labeled in red (up-regulated) and green (down-regulated) in the individual targeted signaling pathway views (Fig. 4). The key positions of five changed metabolites, including $L$-glutamine, $L$-aspartic acid, $N$-acetyl- $L$-aspartate (NAA), $D$-proline, and pyrroline hydroxycarboxylic, in the pathway of arginine and proline metabolism are shown in Fig. 4A.

Six changed metabolites, including $L$-glutamine, aminoimidazole ribonucleotide, ADP, cyclic-AMP, guanosine monophosphate, and guanosine, were involved in purine metabolisms (Fig. 4B). Finally, three differentiated metabolites, including NAA, $L$-aspartic acid, and $L$-glutamine, were also involved in the alanine, aspartate and glutamate metabolism (Fig. 4C). Web-based MSEA is a convenient method for drawing biological inferences from metabolomic data (Xia and Wishart, 2010b). Fig. 5 showed the metabolic sets enrichment overview of the expressed metabolites, which also showed the amino acid metabolism-related metabolites were highly enriched.

\section{DISCUSSION}

Like the genomic and proteomic studies, the metabolomic analysis allows identifying of low molecular weight metabolites that are involved in disease progression (Roessner and Bowne, 2009). Several studies suggested gene expression changes by $\mathrm{Cd}$ in vitro and in vivo (Lee et al., 2016a; Lee et al., 2015; Tokumoto et al., 2011); however, there are very few studies that have evaluated the metabolomic changes due to the $\mathrm{Cd}$ exposure. Our present study was to investigate the long-term effect of urinary metabolites with $\mathrm{Cd}$ exposure in mice. It is 
S.N. Sarma et al.

Table 1. List of identified metabolites that are different between the control and the Cd-treatment groups.

\begin{tabular}{|c|c|c|c|c|c|}
\hline \# & Metabolite & $\begin{array}{l}\text { Retention } \\
\text { time (min) }\end{array}$ & {$[\mathrm{M}-\mathrm{H}]^{-1}$} & $\begin{array}{l}\text { Elemental Composition } \\
\text { (mass error in ppm) }\end{array}$ & Chemical class \\
\hline & Down regulated & & & & \\
\hline 1 & Guanosine & 0.76 & 283.1133 & $\mathrm{C}_{10} \mathrm{H}_{13} \mathrm{~N}_{5} \mathrm{O}_{5}(2)$ & Nucleotide \\
\hline 2 & Oxo-(purinylamino)butanoic acid & 2.81 & 235.1182 & $\mathrm{C}_{9} \mathrm{H}_{9} \mathrm{~N}_{5} \mathrm{O}_{3}(0)$ & Carboxylic acid derivative \\
\hline 3 & D-Proline & 3.70 & 115.0396 & $\mathrm{C}_{5} \mathrm{H}_{9} \mathrm{NO}_{2}(2)$ & Amino acid \\
\hline 4 & Caffeic acid-sulfate & 4.43 & 258.9912 & $\mathrm{C}_{9} \mathrm{H}_{8} \mathrm{O}_{7} \mathrm{~S}(2)$ & Polyphenol sulfate \\
\hline 5 & Vanilic acid-sulphate & 4.49 & 246.9912 & $\mathrm{C}_{8} \mathrm{H}_{8} \mathrm{O}_{7} \mathrm{~S}(0)$ & Polyphenol sulfate \\
\hline 6 & Pyridoxal & 4.50 & 167.0341 & $\mathrm{C}_{8} \mathrm{H}_{9} \mathrm{NO}_{3}(0)$ & Vitamin B6 \\
\hline 7 & 2-Aminoadenosine & 4.76 & 281.1000 & $\mathrm{C}_{10} \mathrm{H}_{14} \mathrm{~N}_{6} \mathrm{O}_{4}(4)$ & Nucleoside derivative \\
\hline 8 & Pyrroline hydroxycarboxylic acid & 4.77 & 129.0553 & $\mathrm{C}_{5} \mathrm{H}_{7} \mathrm{NO}_{3}(0)$ & Amino acid derivative \\
\hline \multirow[t]{2}{*}{9} & Guanosine monophosphate & 5.96 & 363.0179 & $\mathrm{C}_{10} \mathrm{H}_{14} \mathrm{~N}_{5} \mathrm{O}_{8} \mathrm{P}(0)$ & Nucleotide \\
\hline & Up-regulated & & & & \\
\hline 10 & Glutamine & 0.78 & 145.0612 & $\mathrm{C}_{5} \mathrm{H}_{10} \mathrm{~N}_{2} \mathrm{O}_{3}(4)$ & Amino acid \\
\hline 11 & Homoserine & 0.83 & 118.0501 & $\mathrm{C}_{4} \mathrm{H}_{9} \mathrm{NO}_{3}(7)$ & Amino acid derivative \\
\hline 12 & Phenylacetylglycine & 0.93 & 193.0347 & $\mathrm{C}_{10} \mathrm{H}_{1} 1 \mathrm{NO}_{3}(5)$ & Sugar acid \\
\hline 13 & (S)-2-Acetolactate & 1.26 & 132.0283 & $\mathrm{C}_{5} \mathrm{H}_{8} \mathrm{O}_{4}(5)$ & Fatty acid derivative \\
\hline 14 & L-aspartic acid & 1.31 & 133.0136 & $\mathrm{C}_{4} \mathrm{H}_{7} \mathrm{NO}_{4}(1)$ & Amino acid \\
\hline 15 & N-Acetyl-L-aspartate & 1.60 & 175.0231 & $\mathrm{C}_{6} \mathrm{H}_{10} \mathrm{O}_{7}(6)$ & Amino acid derivative \\
\hline 16 & Asp Glu Cys Cys & 2.11 & 467.0926 & $\mathrm{C}_{15} \mathrm{H}_{24} \mathrm{~N}_{4} \mathrm{O}_{9} \mathrm{~S}_{2}(2)$ & Peptide \\
\hline 17 & Phenylalanine & 2.26 & 164.0709 & $\mathrm{C}_{9} \mathrm{H}_{11} \mathrm{NO}_{2}(4)$ & Amino acid \\
\hline 18 & Dehydro-threonate & 2.33 & 115.0030 & $\mathrm{C}_{4} \mathrm{H}_{6} \mathrm{O}_{5}(1)$ & Amino acid derivative \\
\hline 19 & Cys Cys Thr & 2.40 & 324.0712 & $\mathrm{C}_{10} \mathrm{H}_{19} \mathrm{~N}_{3} \mathrm{O}_{5} \mathrm{~S}_{2}(5)$ & Peptide \\
\hline 20 & Cyclic AMP & 2.43 & 328.0447 & $\mathrm{C}_{10} \mathrm{H}_{12} \mathrm{~N}_{5} \mathrm{O}_{6} \mathrm{P}(1)$ & Nucleotide \\
\hline 21 & Glycyl-Phenylalanine & 2.55 & 203.0810 & $\mathrm{C}_{11} \mathrm{H}_{14} \mathrm{~N}_{2} \mathrm{O}_{3}(5)$ & Peptide \\
\hline 22 & 4-Aminohippurate & 2.75 & 194.0443 & $\mathrm{C}_{9} \mathrm{H}_{10} \mathrm{~N}_{2} \mathrm{O}_{3}(1)$ & Hippuric acid \\
\hline 23 & N-Acetylglutamine & 2.95 & 188.0918 & $\mathrm{C}_{7} \mathrm{H}_{12} \mathrm{~N}_{2} \mathrm{O}_{4}(3)$ & Amino acid derivative \\
\hline 24 & $\mathrm{~N}$-Carboxyethyl- $\gamma$-aminobutyric acid & 3.04 & 156.0660 & $\mathrm{C}_{7} \mathrm{H}_{13} \mathrm{NO}_{4}(2)$ & Amino acid derivative \\
\hline 25 & Aconitic acid & 3.13 & 173.0089 & $\mathrm{C}_{6} \mathrm{H}_{6} \mathrm{O}_{6}(1)$ & Carboxylic acid derivative \\
\hline 26 & Isoferuloyl glucuronide & 3.21 & 369.0827 & $\mathrm{C}_{16} \mathrm{H}_{18} \mathrm{O}_{10}(1)$ & Polyphenol glycoside \\
\hline 27 & Aminoimidazole ribonucleotide & 3.41 & 295.1289 & $\mathrm{C}_{8} \mathrm{H}_{14} \mathrm{~N}_{3} \mathrm{O}_{7} \mathrm{P}(0)$ & Nucleotide imadizole \\
\hline 28 & Glutamylcysteine & 3.45 & 250.0713 & $\mathrm{C}_{12} \mathrm{H}_{13} \mathrm{NO}_{5}(2)$ & Peptide \\
\hline 29 & Heptylmalonic acid & 3.56 & 201.1126 & $\mathrm{C}_{10} \mathrm{H}_{18} \mathrm{O}_{4}(2)$ & Carboxylic acid derivative \\
\hline 30 & Oxo-aminovalerate & 3.61 & 131.0705 & $\mathrm{C}_{5} \mathrm{H}_{9} \mathrm{O}_{3} \mathrm{~N}(3)$ & Keto acid derivative \\
\hline 31 & Adenosine diphosphate & 3.64 & 429.0830 & $\mathrm{C}_{10} \mathrm{H}_{15} \mathrm{~N}_{5} \mathrm{O}_{10} \mathrm{P}_{2}(1)$ & Nucleotide \\
\hline 32 & Gly Gly Gly Ser & 3.68 & 275.0998 & $\mathrm{C}_{9} \mathrm{H}_{16} \mathrm{~N}_{4} \mathrm{O}_{6}(4)$ & Peptide \\
\hline 33 & Hydroxydecanedioic acid & 3.94 & 217.1074 & $\mathrm{C}_{10} \mathrm{H}_{18} \mathrm{O}_{5}(3)$ & Fatty acid \\
\hline 34 & Acetyl-phenylalanine & 4.09 & 206.0814 & $\mathrm{C}_{11} \mathrm{H}_{13} \mathrm{NO}_{3}(4)$ & Amino acid derivative \\
\hline 35 & Tryptophan & 4.22 & 204.0660 & $\mathrm{C}_{11} \mathrm{H}_{11} \mathrm{NO}_{3}(4)$ & Amino acid \\
\hline 36 & Carboxymethyllysine & 4.30 & 204.0655 & $\mathrm{C}_{11} \mathrm{H}_{12} \mathrm{~N}_{2} \mathrm{O}_{2}(5)$ & Amino acid derivative \\
\hline 37 & Hydroxytetradecanedioic acid & 4.31 & 273.1705 & $\mathrm{C}_{14} \mathrm{H}_{26} \mathrm{O}_{5}(2)$ & Fatty acid derivative \\
\hline 38 & Dihydroxybenzenesulfonic acid & 4.39 & 188.9870 & $\mathrm{C}_{6} \mathrm{H}_{6} \mathrm{O}_{5} \mathrm{~S}(2)$ & Polyphenol sulfate \\
\hline 39 & Capryloylglycine & 4.98 & 200.1293 & $\mathrm{C}_{10} \mathrm{H}_{19} \mathrm{NO}_{3}(5)$ & Amino acid derivative \\
\hline 40 & Uridine & 5.30 & 244.1542 & $\mathrm{C}_{9} \mathrm{H}_{12} \mathrm{~N}_{2} \mathrm{O}_{6}(3)$ & Nucleotide \\
\hline
\end{tabular}

well known that chronic exposure to Cd causes bone toxicity; however, it is generally considered that Cd-induced osteomalacia is a result of renal tubulopathy (Horiguchi, 2012; Yamanobe et al., 2015). Our results showing moderate kidney damage in these long-term $\mathrm{Cd}$ exposure mice further support this theory. Urine is the end prod- uct of biological metabolism, which can reflect the metabolic changes in the body. Through the UPLC-QTOF-MS measurement, we investigated the metabolic biomarkers of long-term $\mathrm{Cd}$ exposure in urine and identified 40 metabolites that were significantly changed their regulation due to the long-term exposure of $\mathrm{Cd}$. Among all of 
Metabolic exchange in the kidney by long-term Cd exposure

Table 2. Result from pathway analysis with MetPA, restricted to those with more than one hit or with impact $>0.1$.

\begin{tabular}{|c|c|c|c|c|c|c|c|}
\hline Pathway & Total & Expected & Hits & Raw $p$ & $\begin{array}{c}\text { Holm- } \\
\text { Bonferoni } p\end{array}$ & FDR & Impact \\
\hline Arginine and proline metabolism & 44 & 0.81 & 5 & $9.27 \times 10^{-04}$ & $7.60 \times 10^{-02}$ & $4.32 \times 10^{-02}$ & 0.03 \\
\hline Purine metabolism & 68 & 1.25 & 6 & $1.05 \times 10^{-03}$ & $8.53 \times 10^{-02}$ & $4.32 \times 10^{-02}$ & 0.13 \\
\hline Alanine, aspartate and glutamate metabolism & 24 & 0.44 & 3 & $8.60 \times 10^{-03}$ & $6.88 \times 10^{-01}$ & $2.35 \times 10^{-01}$ & 0.34 \\
\hline Aminoacyl-tRNA biosynthesis & 69 & 1.27 & 4 & $3.42 \times 10^{-02}$ & 1.00 & $7.01 \times 10^{-01}$ & 0.00 \\
\hline D-Arginine and D-ornithine metabolism & 4 & 0.07 & 1 & $7.15 \times 10^{-02}$ & 1.00 & $9.77 \times 10^{-01}$ & 0.00 \\
\hline Phenylalanine, tyrosine and tryptophan biosynthesis & 4 & 0.07 & 1 & $7.15 \times 10^{-02}$ & 1.00 & $9.77 \times 10^{-01}$ & 0.50 \\
\hline D-Glutamine and D-glutamate metabolism & 5 & 0.09 & 1 & $8.86 \times 10^{-02}$ & 1.00 & 1.00 & 0.00 \\
\hline Nitrogen metabolism & 9 & 0.17 & 1 & $1.54 \times 10^{-01}$ & 1.00 & 1.00 & 0.00 \\
\hline Vitamin B6 metabolism & 9 & 0.17 & 1 & $1.54 \times 10^{-01}$ & 1.00 & 1.00 & 0.49 \\
\hline Pyrimidine metabolism & 41 & 0.75 & 2 & $1.72 \times 10^{-01}$ & 1.00 & 1.00 & 0.01 \\
\hline Phenylalanine metabolism & 11 & 0.20 & 1 & $1.85 \times 10^{-01}$ & 1.00 & 1.00 & 0.41 \\
\hline Histidine metabolism & 15 & 0.28 & 1 & $2.44 \times 10^{-01}$ & 1.00 & 1.00 & 0.00 \\
\hline beta-Alanine metabolism & 17 & 0.31 & 1 & $2.71 \times 10^{-01}$ & 1.00 & 1.00 & 0.00 \\
\hline Glyoxylate and dicarboxylate metabolism & 18 & 0.33 & 1 & $2.85 \times 10^{-01}$ & 1.00 & 1.00 & 0.13 \\
\hline Citrate cycle (TCA cycle) & 20 & 0.37 & 1 & $3.11 \times 10^{-01}$ & 1.00 & 1.00 & 0.04 \\
\hline Glutathione metabolism & 26 & 0.48 & 1 & $3.85 \times 10^{-01}$ & 1.00 & 1.00 & 0.08 \\
\hline Tryptophan metabolism & 40 & 0.73 & 1 & $5.28 \times 10^{-01}$ & 1.00 & 1.00 & 0.18 \\
\hline Tyrosine metabolism & 44 & 0.81 & 1 & $5.63 \times 10^{-01}$ & 1.00 & 1.00 & 0.00 \\
\hline
\end{tabular}

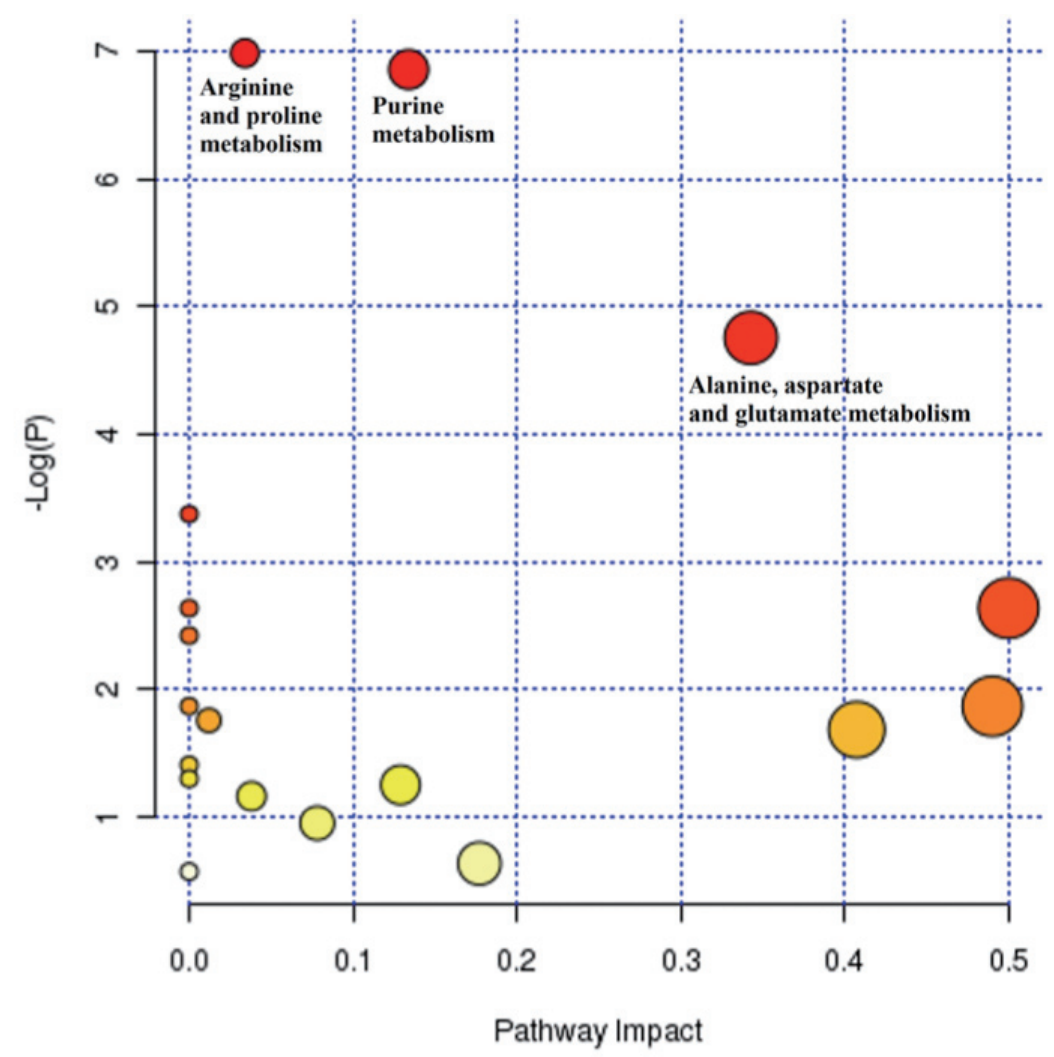

Fig. 3. Summary of ingenuity pathway analysis with MetPA. The pathway impact is calculated as the sum of the importance measures of the matched metabolites normalized by the sum of the importance measures of all metabolites in each pathway. The size of the dot indicates the numbers of matched metabolites in that pathway (bigger dot represents higher matched metabolites number). 
S.N. Sarma et al.
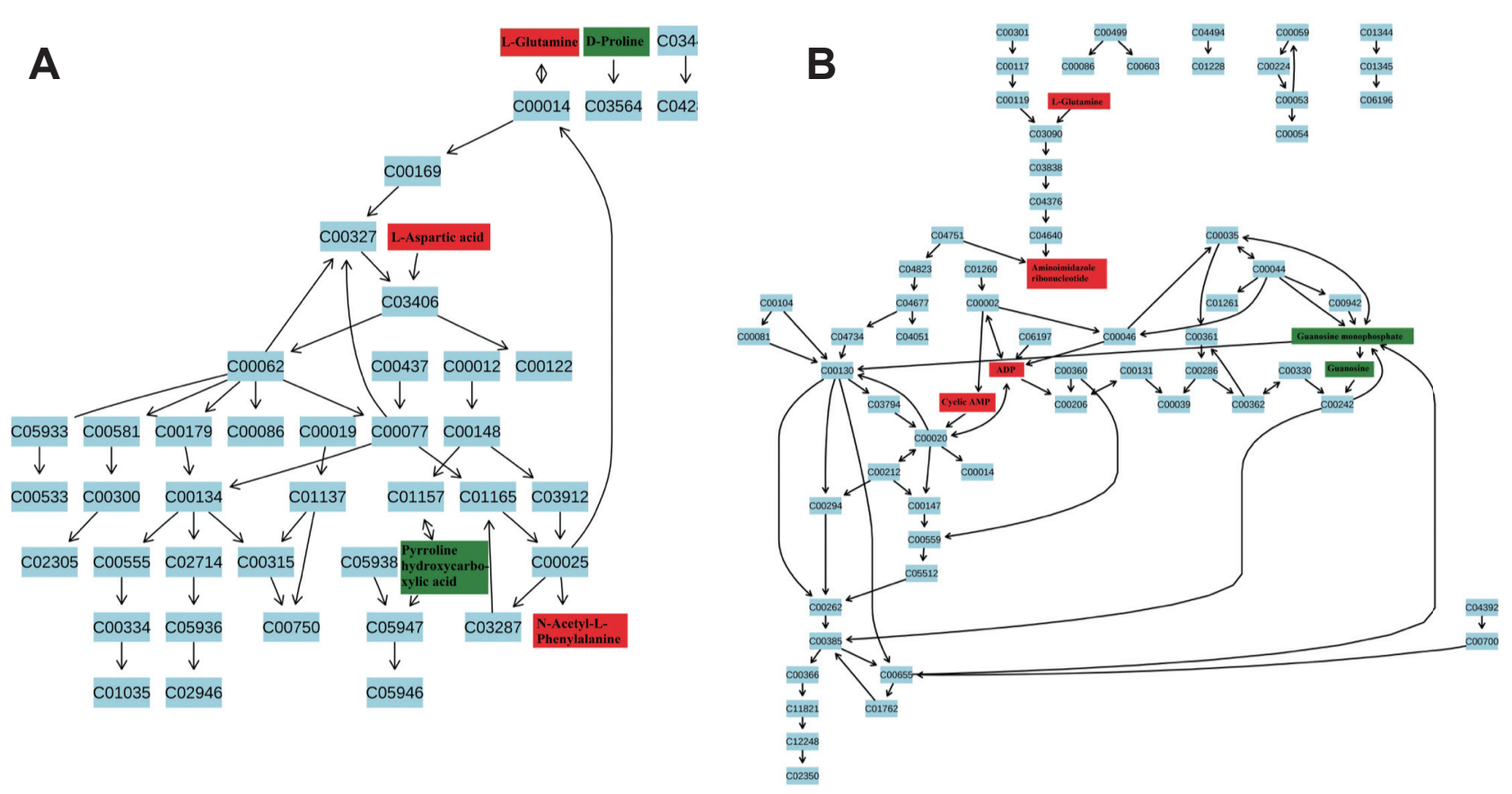

C

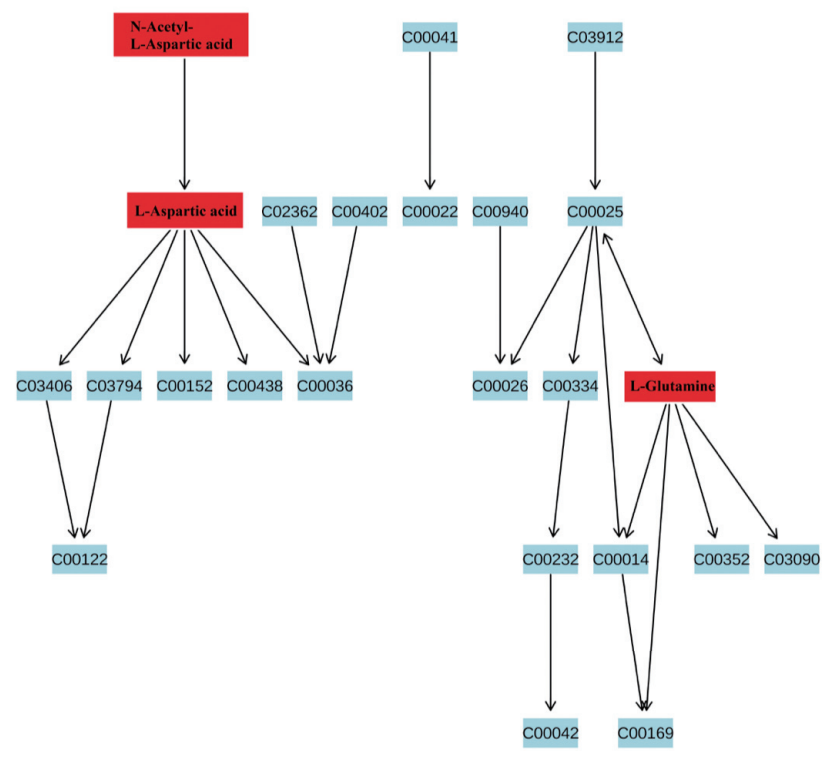

Fig. 4. Pathway diagrams showing the roles of identified metabolites in the arginine and proline metabolism (A); purine metabolism (B); and alanine, aspartate and glutamate metabolism (C). Pathway view shows the significantly changed metabolites in key positions for the pathway. The upstream metabolites will have regulatory roles for the downstream metabolites. Metabolites are presented as code number, and the codes are replaced with name by the significant metabolites found in our study.

the metabolites, most of them are amino acid and their derivatives. The increase of amino acids in the urine is likely an indication of the damage of renal tubules by $\mathrm{Cd}$ resulting in decrease rate of reabsorption. The abnormal amino acid excretion was found in Cd-exposed individu- als (Satarug et al., 2011).

The amino acid glutamine plays a role in a variety of biochemical functions, including protein synthesis, regulation of acid-base balance, as a source of cellular energy, and many other processes. Arginine and proline metab- 
Metabolic exchange in the kidney by long-term Cd exposure

\section{Metabolite Sets Enrichment Overview}

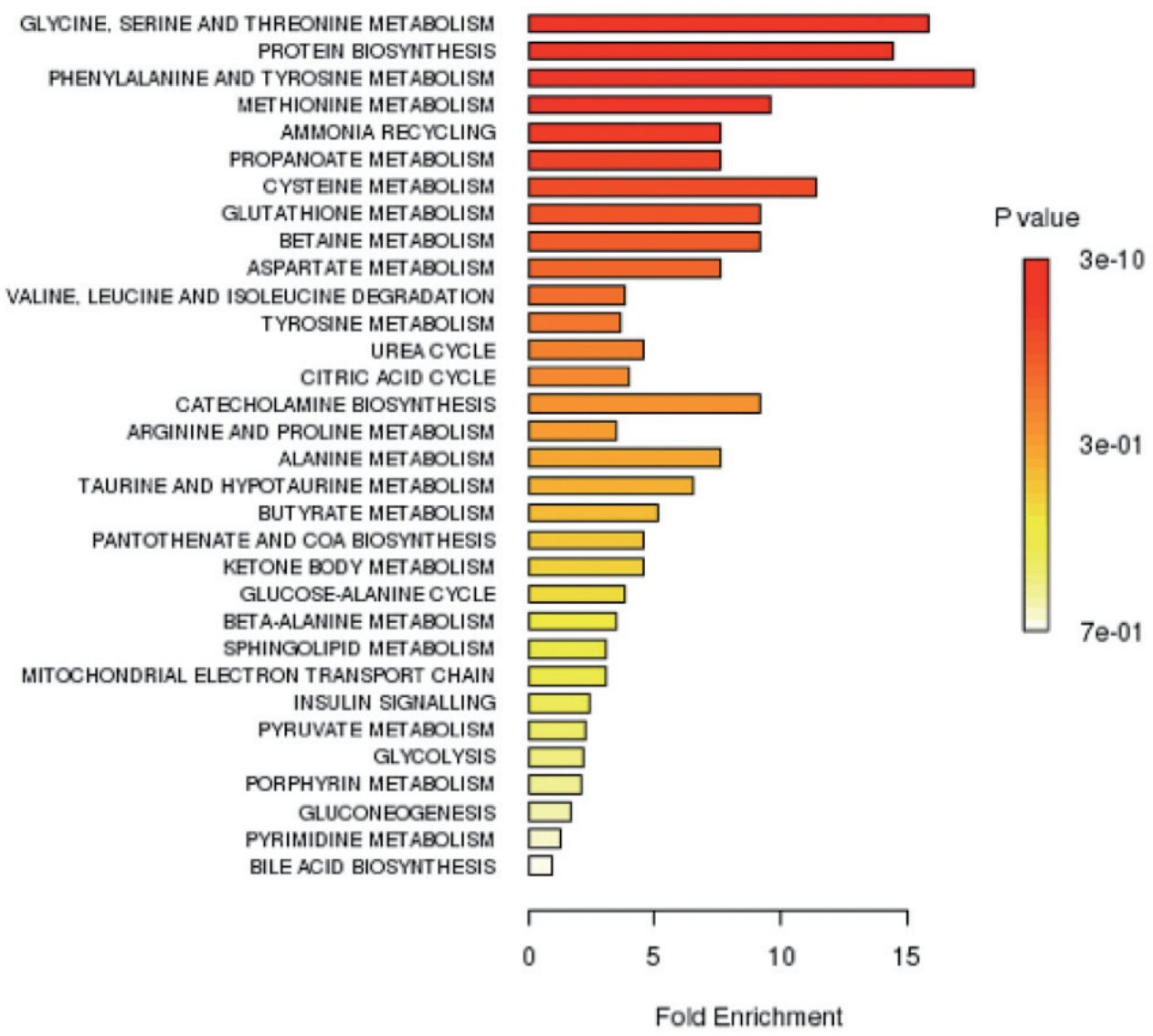

Fig. 5. A summary plot showing the ranking of pathways that are most likely to be affected by long-term Cd exposure.

olism is one of the major bidirectional central pathways (Wu et al., 2009). It has been reported that the glutamine can be up-regulated as a defense of oxidative stress due to long-term Cd expose (Liu et al., 2009). Our metabolic profiling and pathway analysis showed a similar expressional pattern. Purine metabolic pathway is linked to depression and recently found that the high level of blood $\mathrm{Cd}$ is related to the higher depressive symptoms in young adult people (Scinicariello and Buser, 2015). Changes in purine metabolism have been observed in association with low-grade inflammation and increased oxidative stress; both of these are also associated with depression (Kaddurah-Daouk et al., 2013). Cd leads to elevated oxidative stress in mitochondria, which may be associated with depression (Cherkasov et al., 2007). Aminoimidazole ribonucleotide (AIR); an intermediate of purine biosynthesis, adenosine diphosphate, cyclic AMP, guanos- ine and guanosine monophosphate are strongly regulated during the present study. Guanosine was downregulated in patients with major depressive disorder (Ali-Sisto et al., 2016). Glutamate metabolism is linked to aminotransferase reactions in the liver. It increases the transamination of pyruvate to alanine, which is released into circulation and a major contributor of steady-state glutamate synthesis (Kelly and Stanley, 2001). Glutamate has been associated with metabolic programming and subsequent development of the metabolic syndrome may be the consequence of fetal hyperglutamatemia (Sookoian and Pirola, 2012). NAA acid may be involved in energy production from the glutamate in neuronal mitochondria (Clark et al., 2006). $N$-acetyl- $L$-glutamine is slightly less absorbed than glutamine also being up-regulated.

Besides the glutamate metabolic pathway, there are other metabolites related to amino acid metabolism sig- 
nificantly up-regulated due to Cd-exposure. Homoserine is an excreted metabolite produced in the liver from the abnormal methionine production (Gazarian et al., 2002). Increased levels of urinary phenylacetylglycine associated with drug-induced phospholipidosis (Doessegger et al., 2013). (S)-2-Acetolactate is an intermediate in the biosynthesis of valine, leucine, and isoleucine. Phenylalanine is used for protein synthesis or is converted to the nonessential amino acid tyrosine; in addition, kidney plays the metabolic role in this conversation. Phenylalanine hydroxylase is present in the rodent, and human kidney (Møller et al., 2000). Up-regulated phenylalanine may be the result of kidney dysfunction for conversion. 4-Aminohippuric acid is an acyl glycine was significantly increased during Cd-exposure. The measurement of 4-aminohippuric acid in urine can be used to diagnose disorders associated with mitochondrial fatty acid betaoxidation (Li et al., 2016). Furthermore, the increased amount of 4-aminohippuric acid can cause the renal Fanconi syndrome (Hall et al., 2014). $N$-carboxymethyllysine is a product of lipoxidation and glycoxidation, acts as a marker of oxidative stress and long-term damage to proteins (Fu et al., 1996). It is also a biomarker for coronary artery disease and age-related macular degeneration (Stanislovaitienè et al., 2016). Hypertryptophanuria is also a metabolic disorder caused by the massive buildup of tryptophan in the urine (Snedden et al., 1983). The elevated levels of these metabolites may indicate that the long-time Cd-exposure is responsible for the renal dysfunction due to the oxidative stress.

Our findings provide useful information for understanding the metabolomic changes in chronic Cd exposure, using the urine samples. The UPLC-QTOF-MS methodology for metabolomics studies allowed the statistical assessment of the effects caused by the $\mathrm{Cd}$ exposure. The arginine and proline metabolism, purine metabolism, and alanine, aspartate and glutamate metabolism pathways are the major metabolic routes of $\mathrm{Cd}$-induced toxicity. The amino acids glutamine, proline, and tryptophan could be used as biomarkers for long- term Cd-exposure. Figure 5 shows the metabolic relationship among the expressed metabolites. We have reported earlier that this long-term $\mathrm{Cd}$ exposure can trigger mild change in liver function (Lee et al., 2016b), it is, therefore, not feasible to determine whether the metabolic change reported here is from kidney injury specifically; however, it is certain that kidney injury was involved in the metabolic change observed. This study demonstrates that metabolomics is useful to elucidate the metabolic responses and biological effects in early toxicological phase induced by longterm $\mathrm{Cd}$ exposure. However, our sample size is small and required further studies to validate these findings.

\section{ACKNOWLEDGMENT}

The present study was partly founded by the research grant from Institute of Pharmaceutical Life Sciences, Aichi Gakuin University.

SNS was funded by a Natural Science and Engineering Research Council of Canada-CREATE training grant. HMC is supported by the Canada Research Chair Program.

We thank sincerely Mr. Hiromitsu Furukawa for his excellent experimental support.

Conflict of interest---- The authors declare that there is no conflict of interest.

\section{REFERENCES}

Akerstrom, M., Barregard, L., Lundh, T. and Sallsten, G. (2013): The relationship between cadmium in kidney and cadmium in urine and blood in an environmentally exposed population. Toxicol. Appl. Pharmacol., 268, 286-293.

Ali-Sisto, T., Tolmunen, T., Toffol, E., Viinamäki, H., Mäntyselkä, P., Valkonen-Korhonen, M., Honkalampi, K., Ruusunen, A., Velagapudi, V. and Lehto, S.M. (2016): Purine metabolism is dysregulated in patients with major depressive disorder. Psychoneuroendocrinology, 70, 25-32.

Bhattacharyya, M.H., Whelton, B.D., Peterson, D.P., Carnes, B.A., Guram, M.S. and Moretti, E.S. (1988): Kidney changes in multiparous mice fed a nutrient-sufficient diet containing cadmium. Toxicology, 50, 205-215.

Cherkasov, A.A., Overton, R.A. Jr., Sokolov, E.P. and Sokolova, I.M. (2007): Temperature-dependent effects of cadmium and purine nucleotides on mitochondrial aconitase from a marine ectotherm, Crassostrea virginica: a role of temperature in oxidative stress and allosteric enzyme regulation. J. Exp. Biol., 210, 46-55.

Clark, J.F., Doepke, A., Filosa, J.A., Wardle, R.L., Lu, A., Meeker, T.J. and Pyne-Geithman, G.J. (2006): N-acetylaspartate as a reservoir for glutamate. Med. Hypotheses, 67, 506-512.

Doessegger, L., Schmitt, G., Lenz, B., Fischer, H., Schlotterbeck, G., Atzpodien, E.A., Senn, H., Suter, L., Csato, M., Evers, S. and Singer, T. (2013): Increased levels of urinary phenylacetylglycine associated with mitochondrial toxicity in a model of druginduced phospholipidosis. Ther. Adv. Drug Saf., 4, 101-114.

Fels, L.M., Wünsch, M., Baranowski, J., Norska-Borówka, I., Price, R.G., Taylor, S.A., Patel, S., De Broe, M., Elsevier, M.M., Lauwerys, R., Roels, H., Bernard, A., Mutti, A., Gelpi, E., Roselló, J. and Stolte, H. (1998): Adverse effects of chronic low level lead exposure on kidney function--a risk group study in children. Nephrol. Dial. Transplant., 13, 2248-2256.

Forsythe, I.J. and Wishart, D.S. (2009): Exploring human metabolites using the human metabolome database. Curr. Protoc. Bioinformatics., Chapter 14, Unit14.18.

Fowler, B.A. (2009): Monitoring of human populations for early markers of cadmium toxicity: a review. Toxicol. Appl. Pharmacol., 238, 294-300.

Friberg, L. (1984): Cadmium and the kidney. Environ. Health 
Metabolic exchange in the kidney by long-term Cd exposure

Perspect., 54, 1-11.

Fu, M.X., Requena, J.R., Jenkins, A.J., Lyons, T.J., Baynes, J.W. and Thorpe, S.R. (1996): The advanced glycation end product, Nepsilon-(carboxymethyl)lysine, is a product of both lipid peroxidation and glycoxidation reactions. J. Biol. Chem., 271, 9982-9986.

Gao, Y., Lu, Y., Huang, S., Gao, L., Liang, X., Wu, Y., Wang, J., Huang, Q., Tang, L., Wang, G., Yang, F., Hu, S., Chen, Z., Wang, P., Jiang, Q., Huang, R., Xu, Y., Yang, X. and Ong, C.N. (2014): Identifying early urinary metabolic changes with long-term environmental exposure to cadmium by mass-spectrometry-based metabolomics. Environ. Sci. Technol., 48, 6409-6418.

Gazarian, K.G., Gening, L.V. and Gazarian, T.G. (2002): L-Homoserine: a novel excreted metabolic marker of hepatitis B abnormally produced in liver from methionine. Med. Hypotheses, 58, 279-283.

Ginsberg, G.L. (2012): Cadmium risk assessment in relation to background risk of chronic kidney disease. J. Toxicol. Environ. Health A, 75, 374-390.

Gong, P., Chang, X., Chen, X., Bai, X., Wen, H., Pi, S., Yang, W., Wang, L. and Chen, F. (2017): Metabolomics study of cadmium-induced diabetic nephropathy and protective effect of caffeic acid phenethyl ester using UPLC-Q-TOF-MS combined with pattern recognition. Environ. Toxicol. Pharmacol., 54, 80-92.

Hałatek, T., Gromadzińska, J., Wasowicz, W. and Rydzyński, K. (2005): Serum clara-cell protein and beta2-microglobulin as early markers of occupational exposure to nitric oxides. Inhal. Toxicol., 17, 87-97.

Hall, A.M., Bass, P. and Unwin, R.J. (2014): Drug-induced renal Fanconi syndrome. QJM, 107, 261-269.

Hocher, B. and Adamski, J. (2017): Metabolomics for clinical use and research in chronic kidney disease. Nat. Rev. Nephrol., 13, 269-284.

Horiguchi, H. (2012): [Current status of cadmium exposure among Japanese, especially regarding the safety standard for cadmium concentration in rice and adverse effects on proximal renal tubular function observed in farmers exposed to cadmium through consumption of self-grown rice]. Nihon Eiseigaku Zasshi. Japanese Journal of Hygiene, 67, 447-454.

Järup, L. (2002): Cadmium overload and toxicity. Nephrol. Dial. Transplant., 17 Suppl 2, 35-39.

Järup, L. and Akesson, A. (2009): Current status of cadmium as an environmental health problem. Toxicol. Appl. Pharmacol., 238, 201-208.

Järup, L., Hellström, L., Alfvén, T., Carlsson, M.D., Grubb, A., Persson, B., Pettersson, C., Spång, G., Schütz, A. and Elinder, C.G. (2000): Low level exposure to cadmium and early kidney damage: the OSCAR study. Occup. Environ. Med., 57, 668-672.

Kaddurah-Daouk, R., Bogdanov, M.B., Wikoff, W.R., Zhu, H., Boyle, S.H., Churchill, E., Wang, Z., Rush, A.J., Krishnan, R.R., Pickering, E., Delnomdedieu, M. and Fiehn, O. (2013): Pharmacometabolomic mapping of early biochemical changes induced by sertraline and placebo. Transl. Psychiatry, 3, e223.

Kaddurah-Daouk, R., Kristal, B.S. and Weinshilboum, R.M. (2008): Metabolomics: a global biochemical approach to drug response and disease. Annu. Rev. Pharmacol. Toxicol., 48, 653-683.

Kelly, A. and Stanley, C.A. (2001): Disorders of glutamate metabolism. Ment. Retard. Dev. Disabil. Res. Rev., 7, 287-295.

Lee, J.Y., Tokumoto, M., Fujiwara, Y., Hasegawa, T., Seko, Y., Shimada, A. and Satoh, M. (2016a): Accumulation of p53 via down-regulation of UBE2D family genes is a critical pathway for cadmium-induced renal toxicity. Sci. Rep., 6, 21968.
Lee, J.Y., Tokumoto, M., Fujiwara, Y., Hwang, G.W., Lee, M.Y. and Satoh, M. (2016b): Sensitivity of MT-III null mice upon chronic exposure to cadmium. Fundam. Toxicol. Sci., 3, 285-289.

Lee, J.Y., Tokumoto, M., Fujiwara, Y. and Satoh, M. (2015): Involvement of ubiquitin-coding genes in cadmium-induced protein ubiquitination in human proximal tubular cells. J. Toxicol. Sci., 40, 901-908.

Lee, Y.K., Park, E.Y., Kim, S., Son, J.Y., Kim, T.H., Kang, W.G., Jeong, T.C., Kim, K.B., Kwack, S.J., Lee, J., Kim, S., Lee, B.M. and Kim, H.S. (2014): Evaluation of cadmium-induced nephrotoxicity using urinary metabolomic profiles in sprague-dawley male rats. J. Toxicol. Environ. Health A, 77, 1384-1398.

Li, J., Guo, J., Shang, E., Zhu, Z., Zhu, K.Y., Li, S., Zhao, B., Jia, L., Zhao, J., Tang, Z. and Duan, J. (2016): A metabolomics strategy to explore urinary biomarkers and metabolic pathways for assessment of interaction between Danhong injection and lowdose aspirin during their synergistic treatment. J. Chromatogr. B Analyt. Technol. Biomed. Life Sci., 1026, 168-175.

Lim, H., Lim, J.A., Choi, J.H., Kwon, H.J., Ha, M., Kim, H. and Park, J.D. (2016): Associations of Low Environmental Exposure to Multiple Metals with Renal Tubular Impairment in Korean Adults. Toxicol. Res., 32, 57-64.

Liu, J., Qu, W. and Kadiiska, M.B. (2009): Role of oxidative stress in cadmium toxicity and carcinogenesis. Toxicol. Appl. Pharmacol., 238, 209-214.

Møller, N., Meek, S., Bigelow, M., Andrews, J. and Nair, K.S. (2000): The kidney is an important site for in vivo phenylalanine-to-tyrosine conversion in adult humans: A metabolic role of the kidney. Proc. Natl. Acad. Sci. USA, 97, 1242-1246.

Peters, J.L., Perlstein, T.S., Perry, M.J., McNeely, E. and Weuve, J. (2010): Cadmium exposure in association with history of stroke and heart failure. Environ. Res., 110, 199-206.

Prozialeck, W.C. and Edwards, J.R. (2010): Early biomarkers of cadmium exposure and nephrotoxicity. Biometals, 23, 793-809.

Prozialeck, W.C. and Edwards, J.R. (2012): Mechanisms of cadmium-induced proximal tubule injury: new insights with implications for biomonitoring and therapeutic interventions. J. Pharmacol. Exp. Ther., 343, 2-12.

Prozialeck, W.C., VanDreel, A., Ackerman, C.D., Stock, I., Papaeliou, A., Yasmine, C., Wilson, K., Lamar, P.C., Sears, V.L., Gasiorowski, J.Z., DiNovo, K.M., Vaidya, V.S. and Edwards, J.R. (2016): Evaluation of cystatin $C$ as an early biomarker of cadmium nephrotoxicity in the rat. Biometals, 29, 131-146.

Roessner, U. and Bowne, J. (2009): What is metabolomics all about? Biotechniques, 46, 363-365.

Satarug, S., Garrett, S.H., Sens, M.A. and Sens, D.A. (2011): Cadmium, environmental exposure, and health outcomes. Cien Saude Colet., 16, 2587-2602.

Scinicariello, F. and Buser, M.C. (2015): Blood cadmium and depressive symptoms in young adults (aged 20-39 years). Psychol. Med., 45, 807-815.

Snedden, W., Mellor, C.S. and Martin, J.R. (1983): Familial hypertryptophanemia, tryptophanuria and indoleketonuria. Clin. Chim. Acta., 131, 247-256.

Sookoian, S. and Pirola, C.J. (2012): Alanine and aspartate aminotransferase and glutamine-cycling pathway: their roles in pathogenesis of metabolic syndrome. World J. Gastroenterol., 18, 3775-3781.

Stanislovaitienė, D., Žaliūnienė, D., Steponavičiūtè, R., Žemaitienė, R., Gustienè, O. and Žaliūnas, R. (2016): N-carboxymethyllysine as a biomarker for coronary artery disease and age-related macular degeneration. Medicina, 52, 99-103. 


\section{S.N. Sarma et al.}

Tokumoto, M., Fujiwara, Y., Shimada, A., Hasegawa, T., Seko, Y., Nagase, H. and Satoh, M. (2011): Cadmium toxicity is caused by accumulation of $\mathrm{p} 53$ through the down-regulation of Ube $2 \mathrm{~d}$ family genes in vitro and in vivo. J. Toxicol. Sci., 36, 191-200.

Wu, G., Bazer, F.W., Davis, T.A., Kim, S.W., Li, P., Marc Rhoads, J., Carey Satterfield, M., Smith, S.B., Spencer, T.E. and Yin, Y. (2009): Arginine metabolism and nutrition in growth, health and disease. Amino Acids, 37, 153-168.

Xia, J. and Wishart, D.S. (2010a): MetPA: a web-based metabolomics tool for pathway analysis and visualization. Bioinformatics, 26, 2342-2344.

Xia, J. and Wishart, D.S. (2010b): MSEA: a web-based tool to iden- tify biologically meaningful patterns in quantitative metabolomic data. Nucleic. Acids Res., 38, W71-77.

Xu, Y., Wang, J., Liang, X., Gao, Y., Chen, W., Huang, Q., Liang, C., Tang, L., Ouyang, G. and Yang, X. (2016): Urine metabolomics of women from small villages exposed to high environmental cadmium levels. Environ. Toxicol. Chem., 35, 12681275.

Yamanobe, Y., Nagahara, N., Matsukawa, T., Ito, T., Niimori-Kita, K., Chiba, M., Yokoyama, K. and Takizawa, T. (2015): Sex differences in shotgun proteome analyses for chronic oral intake of cadmium in mice. PLoS One, 10, e0121819. 